\title{
La Réinvention du mawsim de Nabî Sâlih. Les territoires palestiniens (1997-2000)
}

Emma Aubin-Boltanski

\section{OpenEdition}

1 Journals

Édition électronique

URL : http://journals.openedition.org/assr/1071

DOI : 10.4000/assr.1071

ISSN : $1777-5825$

Éditeur

Éditions de l'EHESS

\section{Édition imprimée}

Date de publication : 1 juillet 2003

Pagination : 103-120

ISBN : 2-222-96737-6

ISSN : 0335-5985

\section{Référence électronique}

Emma Aubin-Boltanski, « La Réinvention du mawsim de Nabî Sâlih. Les territoires palestiniens

(1997-2000) ", Archives de sciences sociales des religions [En ligne], 123 | juillet - septembre 2003, mis en ligne le 25 octobre 2005, consulté le 20 avril 2019. URL : http://journals.openedition.org/assr/1071 ; DOl : 10.4000/assr.1071 


\section{LA RÉINVENTION DU MAWSIM DE NABî SÂLIH LES TERRITOIRES PALESTINIENS (1997-2000)}

En 1997, l'Autorité palestinienne mise en place dans les Territoires palestiniens depuis 1994, annonce par l'intermédiaire de la presse locale, sa décision de « redonner vie » (ihyâ') (1) à deux fêtes musulmanes qui se déroulent chacune dans des sanctuaires dédiés à des prophètes : les mawsim-s de Nabî Mûsâ (Moïse) et de Nabî Sâlih (prophète Sâlih) (2). La tombe de Moïse est située dans le désert à proximité de Jéricho et celle de Sâlih est au centre d'un village du Nord de Ramallah. Ces deux fêtes sont l'occasion pour les dévots de nouer des liens intimes et à chaque fois particuliers avec les prophètes et, à travers eux, avec Dieu. Elles sont également des grands moments d'exaltation politique et nationaliste. Lorsque l'Autorité palestinienne, par l'intermédiaire de ses ministères des Affaires religieuses et de la Culture, prend en charge l'organisation des festivités, leur caractère nationaliste est considérablement renforcé.

L'objet de cet article sera d'analyser les transformations qui ont affecté le mawsim de Nabî Sâlih qui a été " déplacé » de Ramleh, la ville où il avait lieu à l'origine vers un petit village de Cisjordanie du nom de Nab̂̂ Sâlih (3). Je souhaite notamment démontrer que des sanctuaires tout en se caractérisant par une grande « immobilité » sont habités par une tradition, une mémoire collective sans cesse remaniée, reconstruite et que des tensions peuvent s'établir entre les lieux saints et la tradition éminemment plastique qui se fixe sur eux. Plastique et même manipulable au point d'être littéralement vidée de sa signification religieuse et surinvestie par des symboles politiques et nationalistes. Et ceci au prix d'une grande fragilisation du rituel.

(1) La traduction littérale du terme 'ihyâ" est 'revivification'. La connotation religieuse de ce terme est importante, faisant directement référence à l'œuvre du théologien et philosophe al-Ghazâlî (m. 1111) : Ihyâ' 'ulûm ad-dîn (la revivification des sciences religieuses). Nous avons néanmoins choisi de le traduire par l'expression « redonner vie» ou le mot « renouveau » car, nous le verrons, c'est dans ce sens à la fois plus neutre et plus large que les acteurs du mawsim l'emploient.

(2) Sâlih est un prophète arabe antéislamique non identifiable dans la Bible : VII, 73-79 ; XXVI, 141-158; XXVII, 45-53.

(3) Pour éviter toute confusion entre Nabî Sâlih, le prophète et Nabî Sâlih, le village, nous transcrirons le nom de ce dernier en italique. 


\section{Les tombeaux : une «réalité qui dure »}

Il est aujourd'hui admis que la carte de la Terre sainte musulmane «al-ard al-muqadassa » a été dessinée au moment de la contre-croisade sous l'impulsion de Salâh ad-Dîn al-Ayyûbî (m. 1193) et de Zâhir Baybars (m. 1277). La sainteté de Jérusalem, depuis longtemps fermement établie en islam, dépasse alors le mur d'enceinte de la ville pour s'étendre sur les terres qui l'avoisinent et ceci à travers la multiplication des sanctuaires dédiés à des saints et des prophètes (4). La carte spécifiquement musulmane de la Terre sainte qui en ressort s'est largement imposée à travers deux types d'ouvrages: les "Mérites de la Syrie et de Jérusalem » (Fadâ'il ash-Shâm wa-l-Quds) (5) et les guides de pèlerinage (6). Ces ouvrages et la pratique du territoire qu'ils ont induite ont joué un rôle essentiel dans l'émergence, chez les musulmans, d'une entité territoriale séparée. Entité qui avant d'être politique fut sacrée (7).

À côté des lieux saints majeurs - l'esplanade des mosquées à Jérusalem et le Harâm al-Khalîl (Caveau des Patriarches) à Hébron - qui voient converger des musulmans du monde entier, il existe une multitude de sanctuaires qui font l'objet d'un culte moins large : national, régional ou local et qui forment un réseau extrêmement dense. Il s'agit en général de tombeaux ou cénotaphes protégés par une qubba. Ils ont joué un rôle essentiel dans la formation de la Terre sainte musulmane, et aujourd'hui encore, ils demeurent au cœur de l'identité palestinienne musulmane (8).

Deux ouvrages permettent de mesurer l'ampleur du phénomène aujourd'hui : Mohammadan Saints and Sanctuaries in Palestine (1927) de Tawfiq Canaan, et Tabaqât al-anbiyâ' wa-l-awliyâ' as-sâlihîn fî-l-ard al-muqadassa (Strates de prophètes et de saints en Terre sainte) (1993) de Shukrî 'Arrâf. Ces deux études réalisées à plus de soixante années d'écart sont intéressantes à plusieurs égards. Tout d'abord elles amènent au constat que le culte des saints en Palestine connaît une pérennité extraordinaire. Alors que Canaan prédisait la disparition éminente, « sous l'influence de la culture européenne, des différentes coutumes et superstitions recensées dans (son) ouvrage » (9), Shukrî 'Arrâf introduit son livre en expliquant que «l'homme arabe décidément ne peut se défaire de toutes ses superstitions

(4) Yehoshu'a Frenkel, "Baybars and the sacred geography of Bilâd al-Shâm: A chapter in the Islamization of Syria's Landscape », Jerusalem Studies in Arabic and Islam, no 25, 2001, pp. 153-170.

(5) Emmanuel Sivan, " Le caractère sacré de Jérusalem dans l'Islam aux $12^{\mathrm{e}}$ et $13^{\mathrm{e}}$ siècles », Studia Islamica, $\mathrm{n}^{\circ}$ 27, pp. 149-182, 1967 ; Idem, " The Beginnings of the Fadâ'il al-Quds Literature », Israel Oriental Studies, $\mathrm{n}^{\circ} 1,1971$, pp. 263-271.

(6) Guide 'Alî b. Abî Bakr Al-Harawi (m. 1215), Guide des lieux de pèlerinage, traduit et annoté par Janine Sourdel Thomine, Damas, 1957 ; Mujîr ad-Dîn al-HanBaLi (m. 1520), Al-uns al-jalîl bi târikh al-Quds wa-l-Khalîl. Traduction partielle de Henri Sauvaire, Paris, 1876 ; 'Abd al-Ghanî Al-NABUlÛsî (m. 1731), Al-hadra al-unsiyya fì -r-rihla al-qudsiyya.

(7) Rashid Khalidi, The Palestinian Identity, New York, Columbia University Press, 1997. Alexandre Schölch, Palestine in Transformation 1856-1882, Washington, Institute of Palestine Studies, 1986.

(8) Cf Joseph SADAN sur la compétition entre Damas et Jérusalem concernant les tombeaux de Moïse et le développement de patriotismes locaux autour de ces lieux saints. "Le tombeau de Moïse à Jéricho et à Damas ; compétition entre deux lieux saints principalement à l'époque ottomane ", Revue des études islamiques, $\mathrm{n}^{\circ} 49,1981$, pp. 59-99.

(9) Tawfiq CAnaAn, Muhammad Saints and Sanctuaries in Palestine, Jérusalem, Ariel, 1927, p. 5. 
antéislamiques malgré les tentatives de l'Islam pour les effacer» (10). De plus, alors que Canaan (sans prétendre à l'exhaustivité) base son travail sur 573 sanctuaires disséminés dans tout le territoire de l'Ancienne Palestine mandataire, Shukrî 'Arrâf lui, en recense 2571, pour leur grande majorité des centres de dévotions musulmans.

La plupart des sanctuaires traversent intacts les pires bouleversements et conflits armés. Pendant la guerre de 1948 malgré la destruction massive des villages arabes, de nombreuses tombes de saints ont été préservées. Le sanctuaire de Nabî Mûsâ, par exemple, situé dans un lieu hautement stratégique, près de la frontière jordanienne a été épargné alors que le site s'est transformé à de nombreuses reprises en champs de bataille. Notamment pendant la Première Guerre mondiale et en 1967, lors de la conquête de la Cisjordanie par les Israéliens. L'immense cimetière entourant le sanctuaire témoigne de l'intensité des batailles qui se déroulèrent sur le site (11).

Un historien israélien, Meron Benvenisti, s'est interrogé sur ce phénomène « mystérieux » et l'explique (avec une pointe d'ironie) par le fait que les hommes en charge des démolitions devaient craindre la colère des saints (12). Plus tard, ces sanctuaires ont survécu " en l'état » à une politique systématique de remodelage de l'espace de la Terre sainte Le remplacement des cultures en terrasse par une forêt de pins canadiens entre Jérusalem et Tel-Aviv, les constructions de colonies, sortes d'îlots de petits chalets blancs à toits rouges perchés aux sommets des collines, l'arrachage systématique des oliviers dans certains villages de Cisjordanie sont autant d'exemples de cette profonde refonte du paysage de la Palestine historique. Là encore, des petites qubba-s subsistent par ci-par là, seuls témoins du passé.

De même lorsqu'ils ont été accaparés, appropriés par les vainqueurs à des fins religieuses ou profanes, les sanctuaires ont subi peu de transformations. Deux exemples illustrent bien ce phénomène : le sanctuaire de Nabî Sâlih à Ramleh et le sanctuaire de Nevi Samuel près de Jérusalem. Le complexe religieux de Nabî Sâlih est composé d'un immense minaret de 30 mètres de haut. La municipalité israélienne de la ville se l'est accaparé - les musulmans de la ville n'en ont plus qu'un accès extrêmement limité - et l'a rebaptisé «Tour carrée ». Devenu le symbole de la ville aussi bien pour les Israéliens que pour les Palestiniens, il est partout représenté ; sur les murs de la municipalité, sur les papiers en tête de ses représentants... Le second, un sanctuaire musulman dédié à Samuel composé d'une qubba, d'une mosquée et d'un minaret est devenu un lieu de culte juif important tout en préservant son architecture islamique initiale.

Ainsi en paraphrasant Halbwachs, on peut dire que ces lieux saints forment une société muette et immobile qui contraste fortement avec l'agitation des hommes et les bouleversements de l'histoire (13). C'est en partie sur cette "réalité qui dure » que se fixe la mémoire collective palestinienne.

(10) Shukrî 'ArRAF, Tabaqât al-anbiyâ' wa-l-awliyâa' as-sâlihîn fî-l-ard al-muqadassa, Haifa, Tarshiba, 1993, vol. I, p. 7.

(11) Le cimetière de Nabî Mûsâ (environ 8000 tombes) encadre les ailes est, ouest et nord du maqâm. Son ancienneté et sa taille témoignent de l'importance du culte de Nabî Mûsâ. Tawfiq CanAan, op. cit. p. 9, explique que les bédouins de la tribu 'Idwân y enterraient leurs morts au début du siècle.

(12) Meron Benvenisti, The Sacred Landscape, the Buried History of the Holy Land Since 1948, Berkeley, University of California Press, 2000 p. 281.

(13) Maurice Halbwachs, La mémoire collective, Paris, Albin Michel, 1997, p. 193. 


\section{Les mawsim-s : la tradition en mouvement}

Pendant le mawsim d'avril 2000, à Nabî Mûsâ, une femme de Naplouse vêtue d'une robe traditionnelle brodée, avec une longue bougie à la main m'explique avec enthousiasme la raison de sa venue: " Je suis venue de Naplouse pour visiter Sayyidnâ Mûsâ. C'est Salâh ad-Dîn al-Ayyûbî, le héros qui nous a dit de faire le mawsim chaque année, en ce moment pour défendre la Palestine contre les juifs. Je viens avec des bougies chaque année. Pour faire un vœu. Quand c'est la saison des olives je viens avec de l'huile. Et ici tout le peuple palestinien vient : il y a des gens de Naplouse, d'Hébron, de Jéricho, de partout. De toute la Palestine ! Voilà pourquoi je suis venue ».

Les fêtes qui sont célébrées dans certains sanctuaires de saints et de prophètes sont appelées mawsim, un mot qui à l'origine signifiait « marché » et a évolué dans deux directions différentes : il a d'une part pris le sens de fête religieuse et d'autre part celui de saison (14). D'après Canaan (15), en Palestine, avant 1948, il en existait huit (Nabî Mûsâ (entre Jéricho et Jérusalem) ; Nabî Rubin (Jaffa) ; Nabî Sâlih (Ramleh ; le village et Acre) ; Darûm et al-Muntar (Gaza) ; Nabî Yûnis (Sidon). Ils étaient concentrés sur le littoral et dans le centre du pays, autour de Jérusalem. La plupart se déroulaient dans des sanctuaires dédiés à des prophètes. Aujourd'hui, seuls deux de ces mawsim-s sont encore célébrés (Nabî Mûsâ ; Nabî Sâlih) (16).

La date de ces fêtes est fixée en fonction de la Pâques orthodoxe; elles ont donc lieu pendant le mois d'avril. Différentes théories ont été élaborées pour expliquer que des célébrations musulmanes sont ainsi calées sur le calendrier chrétien. Des orientalistes y ont vu la trace persistante de fêtes païennes du printemps (17). Une explication que l'on retrouve essentiellement dans l'historiographie israélienne invoque l'opportunisme politique des Turcs qui auraient créé les mawsim-s à la fin $\mathrm{du} \mathrm{XIX}^{\mathrm{e}}$ siècle pour contrebalancer le poids excessif des pèlerins chrétiens au moment des fêtes de Pâques (18). Une troisième explication, très répandue chez les historiens palestiniens, fait remonter cet opportunisme politique au XII ${ }^{\mathrm{e}}$ siècle et à Salâh ad-Dîn qui aurait créé les mawsim-s pour protéger la Terre sainte contre de nouvelles croisades (19).

Comme on l'a vu avec la vieille dame de Naplouse, la théorie liant la fondation des mawsim-s au grand héros de l'histoire musulmane ne se cantonne pas aux livres d'histoire, elle a aujourd'hui valeur de mythe fondateur du lieu saint. La vieille dame de Naplouse le disait: Aujourd'hui comme hier c'est sur ordre de Salâh ad-Dîn que les Palestiniens se rendent à Nabî Mûsâ, pour défendre « la Palestine »,

(14) Arent Jan Wensick, « mawsim », Encyclopédie de l'Islam (nouvelle édition), Paris, Maisonneuve et Larose 1991.

(15) Tawfiq CanaAn, op. cit., 1927, p. 299.

(16) Aujourd'hui, les Druzes célèbrent le mawsim de Nabî Shu'ayb dans un village situé aux alentours de Tibériade et les Palestiniens d'Israël le mawsim de 'Alî b. Sinan, à Hertzeliyya. Mais ces deux fêtes ne sont pas mentionnées par Tawfiq Canaan.

(17) Arent Jan Wensick, op. cit; Gustav Von Grunebaum, Muhammadan Festivals, Londres, Abelard-Schuman, 1976.

(18) Yehoshuah Ben ARIen, The Rediscovery of the Holy Land in the $19^{\text {th }}$ Century. Jérusalem, Israel Exploration Society, 1979, p. 151.

(19) Umar Sâlih BARGHuthi, Târikh Filastîn, Jérusalem, Filastîn, 1923 ; Kamel al-'AsAli, Mawsim an-Nabî Filastîn, Mûsâ, 'Ammân, al-Jâmi’a al-Urdûniyya, 1990. 
reproduisant ainsi la geste du vainqueur de Jérusalem et se plaçant dans une longue lignée politico-croyante remontant au XII ${ }^{\mathrm{e}}$ siècle.

En réalité, cette théorie ne s'appuie sur aucune source historique. Le sanctuaire de Nabî Mûsâ a été construit à l'époque de Zâhir Baybars, près de cent ans après la mort de Salâh ad-Dîn. Quant à celui de Nabî Sâlih, la première mention qui en est faite dans les sources arabes remonte au XVe siècle (20). Baybars a bien assigné des biens de main morte (waqf) au sanctuaire de Nabî Mûsâ pour son entretien et l'accueil des pèlerins mais il n'existe aucune mention concernant la tenue d'une fête annuelle antérieure au $\mathrm{XV}^{\mathrm{e}}$ siècle (21). Dans les textes ultérieurs que j’ai pu réunir (couvrant trois siècles : $\left.X_{V I I}{ }^{\mathrm{e}}-\mathrm{XIX}^{\mathrm{e}}\right)(22)$, il n'est fait aucune mention de Salâh ad-Dîn ou d'une quelconque relation avec les croisades. Il s'agit donc d'une « tradition inventée » dont la genèse peut être facilement retracée. Il semble en effet qu'elle ne commence à apparaître qu'aux alentours des années 1920 sous la plume d'historiens et folkloristes palestiniens (23) et qu'elle soit directement liée à l'émergence du mouvement nationaliste palestinien.

Les années 1920 constituent une période charnière dans l'histoire de la formation de l'identité palestinienne. Elles marquent le début du Mandat britannique ; les nationalistes palestiniens prennent alors des distances avec la Grande Syrie et opèrent un recentrage sur la Syrie du Sud et c'est également à ce moment qu'apparaît le grand leader du mouvement, Hajj Amîn al-Husaynî. Dans ce contexte, les deux mawsim-s de Nabî Mûsâ et de Nabî Sâlih (à Ramleh) deviennent des occasions de grandes manifestations nationalistes menées par les leaders de l'époque. Bien plus, alors que jusque-là ils n'attiraient que des musulmans issus de régions particulières - les habitants de la côte pour le mawsim de Nabî Sâlih et ceux des régions montagneuses du centre pour celui de Nabî Mûsâ - ils voient dorénavant converger des gens venus de l'ensemble du territoire en " délégations » derrière les drapeaux des confréries et de plus en plus derrière les bannières des différentes villes ou villages représentés. Ce qui fait dire à de nombreux historiens (24) que les mawsim-s ont joué un rôle essentiel dans l'émergence d'une identité palestinienne. D'après eux, ces fêtes qui attiraient chaque année des fidèles musulmans venus de toute la Palestine auraient permis de tisser des liens entre les différentes régions du pays et auraient contribué à forger un sentiment d'appartenance à une identité commune.

Ces deux lieux de pèlerinage «secondaires » (25) sont devenus les centres d'une intense activité politique et nationaliste à une époque où le culte des saints était critiqué et attaqué par les réformistes dont l'influence était importante sur les

(20) Mujîr ad-Dîn al-HanBaLi, Al-uns al-jalîl bi târikh al-Quds wa-l-Khalîl, Baghdad, Maktaba an-Nahda, 1995, vol. 2. p. 69.

(21) Henri Sauvaire, Histoire de Jérusalem et d'Hébron. Fragments de la chronique de Mudjir ad-Dîn, Paris, Leroux, 1876, pp. 26-27.

(22) Sur les auteurs évoquant le mawsim de Nabî Mûsâ, cf la bibliographie très complète de Kamel al-'ASALî, op. cit. et Joseph SADAN, op. cit. L'un et l'autre ont réuni une cinquantaine d'auteurs couvrant trois siècles : $\mathrm{XVII}^{\mathrm{e}}-\mathrm{XIX}{ }^{\mathrm{e}}$ siècles. Concernant le mawsim de Nabî Sâlih, en revanche, les sources manquent cruellement.

(23) Le premier historien à mentionner un lien entre Salâh ad-Dîn et les mawsim-s est Umar Sâlih BARghÛthî, dans son histoire de la Palestine éditée à Jérusalem en 1923, op. cit.

(24) Rashid Khalidi, op. cit. p. 151 ; Kamel al-'Asali, op. cit. Alexander SchÖlch, op. cit, p. 16 ; Yehoshuah Porath, The Emergence in the Palestinian-arab National Movement, 1919-1929, Londres, Frank Cass, 1973, pp. 1-9.

(25) Niels Henrik Olesen, Culte des Saints et Pèlerinages chez Ibn Taymiyya, Paris, Geuthner, 1991. 
milieux nationalistes arabes (26). Les grands leaders, comme Hajj Amîn al-Husaynî ont été sensibilisés aux doctrines de Muhammad 'Abduh (1849-1905) et surtout de Rachid Rida (1865-1935) (27). On peut donc supposer que ces deux fêtes religieuses populaires n'auraient pas joué ce rôle de catalyseur nationaliste sans leur inscription dans la grande histoire musulmane par le biais de la figure de Salâh ad-Dîn al-Ayyûbî.

Cet aperçu historique permet de comprendre comment une nouvelle figure peut s'imposer au centre d'une fête religieuse dans un contexte politique et social particulier. Les prophètes, Moïse et Sâlih semblent relégués au second plan au profit du grand héros de l'histoire musulmane qui est mieux à même de répondre aux attentes du présent et constitue un espoir pour le futur. Mais les prophètes ne disparaissent pas pour autant. Ils deviennent eux-mêmes des mujâhid-s, apparaissant habillés de vert et brandissant des épées dans les songes des pèlerins ou aux alentours de leurs tombeaux. C'est particulièrement le cas du prophète " arabe »Sâlih qui, à la différence de Moïse, a une identité floue et est fréquemment assimilé à un homme pieux qui à l'époque de la contre-croisade combattit auprès de Salâh ad-Dîn (28).

Les sanctuaires sont «cette réalité qui dure » caractérisée par l'immobilité. Mais la tradition qui les place en son centre, qui se fixe sur eux est, au contraire, constamment reformulée. Les mawsim-s sont devenus des modes d'action politique dans les années 1920 et sont acceptés et même revendiqués comme tels par l'ensemble des acteurs, qu'ils soient organisateurs ou simples pèlerins. L'événement fondateur est lui-même sujet à rélaboration alors même qu'il est constitué comme référence immuable. Et c'est là toute la difficulté pour celui qui se propose d'étudier "l'activité religieuse en train de se faire » (29): alors que les acteurs affirment que leurs pratiques et représentations religieuses sont inchangées depuis des temps immémoriaux, une analyse dans la diachronie permet de constater qu'elles sont au contraire d'une grande plasticité et qu'elles évoluent à travers le temps.

La partie qui suit sera consacrée au mawsim de Nabî Sâlih entre 1997 et 2000 et permettra d'illustrer notre propos sur la tension entre lieu saint «tyrannique » et tradition. Pour comprendre son évolution, il sera nécessaire d'évoquer en contrepoint, le mawsim de Nabî Mûsâ. Ces deux fêtes présentent de nombreuses caractéristiques communes mais pendant la période qui m'intéresse (1997-2000), elles se déroulaient de façons très différentes. Leurs dissemblances s'expliquent en grande partie par leur histoire particulière. Alors que le pèlerinage de Nabî Mûsâ a connu une continuité extraordinaire - même aux pires moments de l'histoire palestinienne, des fidèles ont continué à respecter la tradition de se rendre sur le tombeau de Moïse - celui de Nabî Sâlih a été interrompu à de nombreuses reprises et, bouleversement sans précédent, en 1997, l’Autorité palestinienne a décidé de « le dépla-

(26) Albert Hourani, Histoire des peuples arabes, Paris, Seuil, 1990, pp. 403-416.

(27) Philip. Mattar, The Mufti of Jerusalem, New York, Columbia University Press, 1988, pp. 9 ; 12 : Hajj Amin a fait plusieurs séjours au Caire pour suivre une formation religieuse. Rashid Rida était un ami de la famille.

(28) Walîd RABî'A, « Mawsim an-Nabî Sâlih », Al-Turâth wa-l-mujtama', 1985, nº 25, pp. 85-90. Pour l'auteur de cet article, il ne fait aucun doute que Sâlih était un soldat de Salâh ad-Dîn ou le neveu du Prophète Muhammad.

(29) Albert Piette, La religion de près, l'activité religieuse en train de se faire, Paris, Métaillé, 1999. Les difficultés rencontrées sur le terrain musulman ont été analysées par Dale F. EicKELMAM et James PIsCatori, Muslim Travellers, Berkeley, University of California Press, 1990. 
cer» de la ville de Ramleh où il se déroulait traditionnellement pour «le ressusciter " dans un village de Cisjordanie appelé Nabî Sâlih.

La continuité du mawsim de Nabî Mûsâ a été assurée grâce aux liens individuels que des fidèles (des femmes surtout mais également des mystiques) ont toujours pu entretenir avec Moïse à travers des rituels de vœux (nidhr) (30), d'invocations (da'wa), de circoncision ou de coupe de cheveux des jeunes garçons ( 'aqî $q a) . .$. À partir du moment où le ministère palestinien des Affaires religieuses a décidé de « ressusciter le mawsim »-1'emploi de cette expression est significatif de la volonté d'ignorer la formidable permanence du rituel - le sanctuaire s'est transformé en une " arène » où différents discours et pratiques rivalisaient. La principale tension se situait entre une pratique rituelle intime et à chaque fois particulière dont le tombeau de Moïse était le centre et une pratique collective d'exaltation de la nation et de son leader Yasser Arafat orchestrée par des représentants du pouvoir politique. Malgré la volonté des organisateurs de contrôler les festivités en instaurant un programme et des règles de conduite précises, le mawsim se déroulait dans un chaos indescriptible.

À Nabî Sâlih, comme nous le verrons plus en détail, la situation était tout à fait différente. Le mawsim n'avait plus lieu depuis dix ans et l'Autorité palestinienne, depuis qu'elle l'avait "déplacé » et "réinventé » en contrôlait parfaitement le déroulement.

\section{La réinvention du mawsim de Nabî Sâlih (1997-2000)}

\subsection{Les mawims-s de Nabî Sâlih}

Huit sanctuaires sont dédiés au prophète Sâlih en Palestine. D'après Tawfiq Canaan, ils jouaient un rôle central dans le dispositif de protection de la Palestine mis en place par Salâh ad-Dîn (31). Au début du siècle trois d'entre eux étaient le centre de mawsim-s au moment des fêtes de Pâques (Acre, le village de Nabî Sâlih et Ramleh). En réalité dans la mémoire collective, le mawsim de Nabî Sâlih est associé à la ville de Ramleh. C'est dans cette ville située non loin de la côte, que les festivités étaient les plus importantes. Dans le village de Nab̂̂ Sâlih et à Acre, des rassemblements étaient bien organisés pour célébrer Nabî Sâlih mais ils n'avaient pas le caractère massif de celui de Ramleh pour lequel des fidèles venus de l'ensemble de la Palestine se déplaçaient. Il est également généralement admis que Sâlih ne fit que "prier et passer » dans le village de Nabî Sâlih et à Acre et que sa "vraie tombe » se trouve à Ramleh. Les différents sanctuaires dessinent le parcours que le prophète aurait suivi avant de s'arrêter à Ramleh.

(30) En arabe dialectal palestinien, le mot 'nadhr' se prononce 'nidhr'.

(31) Tawfiq Canaan reprend la théorie selon laquelle les mawsim-s ont été créés pour contrer de nouvelles croisades en Terre sainte. Mais selon lui c'est Baybars et non pas Salâh ad-Dîn qui serait à l'origine de cette stratégie de défense, op. cit. p. 299. 
Au lendemain de la guerre de 1948, Ramleh pratiquement vidée de sa population arabe est devenue une agglomération israélienne importante. Le mawsim a été interrompu et repris à plusieurs reprises pour être finalement définitivement interdit en 1988, au début de la première Intifada. La même année, le complexe religieux a été classé monument historique et son accès est devenu payant. Les musulmans de la ville et des alentours ne peuvent s'y rendre que le vendredi matin, seul moment de la semaine où il leur est ouvert. Cela dit, le sanctuaire est toujours le centre d'une intense activité rituelle et surtout étant toujours « là », constituant cette réalité qui dure, il conserve sa place de lieu de mémoire pour les Palestiniens (32).

Dans ce contexte, la décision de l'Autorité palestinienne de ressusciter le mawsim en le déplaçant dans le village de Nabî Sâlih est apparue comme un véritable bouleversement. Et même si le mawsim réinventé a été un succès, le souvenir de celui que certains appellent "an-Nabî Sâlih al-haqîq $̂$ » (le vrai Nabî Sâlih), faisant ainsi référence au sanctuaire de Ramleh, restait très présent suscitant des heurts entre visiteurs et organisateurs. Cette tension entre lieu saint (33) et tradition qui tente de s'en libérer est en grande partie à l'origine de l'effacement progressif du prophète des festivités telles qu'elles ont été « réinventées » en 1997 par l'Autorité palestinienne. Festivités qui, nous le verrons, d'année en année perdront leur signification religieuse.

\subsection{L'appel à participer au mawsim de Nabî Sâlih}

À partir de janvier 1997, des articles ont commencé à paraître dans la presse palestinienne sur le mawsim de Nabî Sâlih et la décision de reprendre cette tradition dans le cadre de la "résurrection du patrimoine palestinien ». Le ministère de la Culture s'est vu confier la tâche d'organiser les festivités et un " Haut comité pour la reprise, le renouveau du mawsim de Nabî Sâlih " (Lajna 'ulya li-i'âdat ihyâ' mawsim an-Nabî Sâlih) a été créé pour rassembler les fonds nécessaires et diffuser le plus largement possible en Cisjordanie «l'invitation au peuple palestinien à participer aux festivités ». Le directeur du Comité s'est déplacé de ville en ville pour faire des conférences sur le mawsim. Invariablement il expliquait que "c'est Salâh ad-Dîn qui a imaginé ces rassemblements pour contrer toute nouvelle attaque des Croisés grâce à la mobilisation (tajnîd) de très nombreuses forces vives. » Il ajoutait qu'avant 1937, Hajj Amîn lui-même « était derrière ces festivités et en tirait un profit politique » et concluait en ces termes : «Les Palestiniens doivent poursuivre l'œuvre de Salâh ad-Dîn et de Hajj Amîn pour défendre la Palestine contre l'occupation et l'extension des colonies. Pour cette raison, le ministère de la Culture a décidé de ressusciter le mawsim de Nabî Sâlih après soixante ans d'interruption. » Ainsi, tout en mobilisant la grande histoire musulmane et nationale à aucun

(32) Cf. Maurice Halbwachs, op. cit, p. 196. Il explique très bien que si l'on veut détruire une communauté, rien n'est fait tant que l'on n'a pas détruit les murs, la pierre... et que les gens qui en garde la mémoire sont toujours en vie.

(33) Catherine Mayeur-Jaouen «Tombeau, mosquées et zâwiya; La polarité des lieux saints musulmans » in André Vauchez, éd., Lieux sacrés, lieux de culte, sanctuaires, Rome, École Française de Rome, 2000, pp. 133-147. Pour l'auteur la « dictature » du lieu saint en islam s'explique par l'importance des tombeaux dans le culte des saints musulmans. Importance due à l'absence (contrairement au christianisme) de représentations du saint et de reliques. 
moment il n'évoquait Ramleh. Tout était fait pour donner l'impression que le mawsim s'était toujours déroulé dans le village.

La décision de l'Autorité palestinienne a été vivement critiquée, notamment par les réfugiés originaires des villes côtières qui y ont vu un renoncement aux Territoires de 1948. Elle a été interprétée comme un défi lancé à la "vieille Palestine » (34) et une tentative de définir de nouvelles frontières, de dessiner une "nouvelle Palestine », conforme au processus de paix. Décision courageuse et risquée qui n'a en fait jamais été assumée dans ces termes par les organisateurs qui ont su démontrer que leur choix loin d'être une abdication s'inscrivait au contraire dans le cadre de la "lutte nationale pour la terre » et qu'en réunissant des gens venus de l'ensemble de la Palestine, c'était "la nation et la terre palestiniennes » qui étaient célébrées. C'est sous la forme d'un second défi qu'ils préféraient défendre leur projet. Un défi lancé à l'émiettement du territoire palestinien consécutif aux accords d'Oslo. Les accords d'Oslo ont en effet entériné une double division du territoire qui constitue le référent identitaire des Palestiniens ('Ancienne Palestine mandataire) : une première en quatre grands blocs, entre Israël, Jérusalem, la Bande de Gaza et la Cisjordanie et une seconde qui concerne la Cisjordanie en trois zones différentes (A, B et C) (35). Cet «émiettement» du territoire a eu des conséquences sur le corps social qui n'a jamais retrouvé son unité. En fonction de leur lieu d'habitation, les Palestiniens étaient en effet soumis à des régimes administratifs particuliers et avaient des capacités de mobilité différentes. C'est donc dans ce contexte de fragmentation territoriale et sociale que l'Autorité palestinienne a décidé de reprendre le mawsim. Une fête qui devait permettre de réunir dans un temps et un espace limités l'ensemble des composantes de la société palestinienne.

Le village de Nabî Sâlih qui accueille désormais le mawsim "ressuscité » est situé à une vingtaine de kilomètres au nord de Ramallah, dans la plaine de Bani Zeid. Il a 400 habitants. Il constitue un parfait exemple de l'éclatement du territoire palestinien. Avant 1948, il était le centre d'un pèlerinage local lié au grand mawsim de Ramleh. Les habitants de la plaine de Bani Zeid (13 villages) s'y rassemblaient pour ensuite partir en procession vers Ramleh situé à $25 \mathrm{~km}$ plus à l'ouest et participer au mawsim. Les liens entre la ville et les villages de la plaine étaient importants et anciens (Mujir ad-Dîn al-Hanbali en fait état au $\mathrm{XV}^{\mathrm{e}}$ siècle) (36). Le mawsim constituait un moment central dans la vie des paysans ; ils se déplaçaient chaque année à Ramleh pour des raisons économiques et surtout religieuses (visites pieuses au prophète, sacrifice du mouton, circoncision...). Depuis 1948, la ligne verte, la frontière entre Israël et les territoires palestiniens les sépare et les villageois n'entretiennent plus les mêmes liens avec la ville qui pour eux est désormais extrêmement lointaine : pour se rendre à Ramleh, il faut passer par Ramallah, Jérusalem et ensuite remonter vers le nord-ouest par la route de Tel-Aviv.

À cette coupure déjà ancienne s'en est ajoutée une autre. Depuis les accords d'Oslo, la plus grande partie de la plaine de Banî Zeid a été placée en zone A, sous administration civile et sécuritaire palestinienne. Le village de Nabî Sâlih, quant à lui, a été classé en zone $\mathrm{B}$ où les Palestiniens détiennent le pouvoir civil et les

(34) Meron Benvenisti, op. cit. p. 281.

(35) Zone A (zone autonome palestinienne); Zone B (zone sous responsabilité partagée palestinienne et israélienne); Zone C (zone demeurant sous administration israélienne)

(36) Mujîr ad-Dîn al-HanbaLî, op. cit. p. 230 
Israéliens continuent d'être en charge de la sécurité générale. Et ceci parce qu'il est situé en face de Halamish, une colonie israélienne construite entièrement sur ses terres.

\subsection{Le clan, le folkloriste et le prophète}

Les liens que le village entretenait avec la ville de Ramleh sont à l'origine du choix d'organiser le pèlerinage dans ce nouveau cadre. Trois autres éléments ont largement contribué au succès du projet : le clan familial habitant le village, le folkloriste local et la présence d'un sanctuaire dédié au prophète Sâlih.

La mainmise de l'Autorité palestinienne sur le mawsim de Nabî Sâlih s'est faite par l'intermédiaire d'un clan familial important et puissant très représenté au sein de l'Autorité : celui des Tamîmî. Le succès du mawsim "réinventé » à Nabî Sâlih est en grande partie dû au rôle joué par cette famille qui a su faire valoir sa légitimité historique et religieuse pour asseoir son autorité sur le déroulement des festivités. Les habitants de Nab̂̂ Sâlih sont fiers d'affirmer qu'ils sont «tous Tamîmî ici, qu'ils forment une seule et même famille ». Au centre du village se trouve le diwân familial récemment restauré et agrandi. Au cours d'une discussion sur la restauration de ce bâtiment, un villageois m'a fait cette remarque : " Nous n'avons jamais été autant Tamîmî, avant on ne disait pas : 'Je suis Tamîmî' mais je suis Abû Fulân (untel) de la famille, Hamdan, Ayyûb, 'Amara ou Shîdî du village de Nabî Sâlih. Avant ça n'avait pas de sens de dire : 'Je suis Tamîmî' parce qu'il y a des millions de Tamîmî dans le monde. Maintenant on ne dit que ça : 'Nous sommes Tamîmî' ". Se dire Tamîmî c'est s'inscrire dans la descendance de Tamîm ad-Dârî, le Compagnon du Prophète Muhammad (m. 660). La personnalité de Tamîm ad-Dârî, chrétien de Palestine converti très tôt à l'islam permet au groupe de revendiquer une double légitimité : religieuse et nationale. De plus, être Tamîmî, c'est avoir été désigné directement par le Prophète et donc par Dieu pour protéger la Terre sainte, la Palestine. La tradition veut en effet que le Prophète Muhammad lui-même ait désigné Tamîm ad-Dârî comme gouverneur d'Hébron et comme responsable des waqf-s de la ville (37). Comme le laissait entendre le villageois cité plus haut cette double légitimité sans cesse mise en avant - en particulier au moment des festivités - est récente.

La présence dans le village du folkloriste palestinien contemporain le plus connu, Nimr Sirhan, a joué un rôle essentiel dans la reprise du mawsim. Outre une trentaine d'ouvrages sur les chants, les costumes, les légendes palestiniennes, il est l'auteur d'une encyclopédie en trois volumes sur le folklore palestinien. Selon ses propres termes, il a théorisé "l'instrumentalisation politique et nationaliste du patrimoine palestinien dans la lutte contre l'occupant israélien ». De 1967 à 1995, il a vécu en exil en Jordanie. De retour en Palestine, il s'est installé dans le village de Nabî Sâlih d'où sa femme est originaire. Il est employé par le ministère de la Culture. Pendant les trois premières années, il a dirigé le Haut Comité pour le renouveau du mawsim et a écrit une centaine d'articles sur les festivités parus dans les deux principaux quotidiens palestiniens, al-Ayyâm et al-Quds. Son discours sur

(37) Sur cette tradition, cf. Fritz KRENKow, «The Grant of Land by Muhammad to Tamîm ad-Dârî », Islamica, no 1, 1925, pp. 529-32. David Cook, "Tamîm ad-Dârî », Bulletin of the School of Oriental and African Studies, ${ }^{\circ}$ 61, 1998, pp. 20-28. 
les mawsim-s et les tombeaux des saints reflète les sentiments multiples et contradictoires qui entourent le culte des saints en Palestine à la fois condamné par une certaine orthodoxie et dans le même temps élevé au rang de pilier de l'identité nationale. Il se définit comme un fervent musulman moderne, fait la prière cinq fois par jour, le jeûne chaque année et espère se rendre bientôt en pèlerinage à la Mecque. Il s'élève contre ceux qui "croient » que Nabî Sâlih est enterré dans le village ou ailleurs en Palestine et vouent un culte au prophète car «les prières doivent être destinées à Dieu seul » et que toutes ces croyances ne sont que "innovations» (bid'a), «illusions» (awhâm) ou «imitations des chrétiens». Dans le même temps, il soutient que les traditions populaires doivent être perpétuées parce qu'elles constituent le cour de l'identité palestinienne. Et c'est sans doute pour cette raison, qu'en mai 1997, juste après le début des festivités, il n'a pas hésité à rendre public - par l'intermédiaire de la presse locale (38) - un événement dont il a été l'acteur ; le prophète Sâlih lui est apparu en songe habillé de vert, l'épée à la main pour le remercier d'avoir ainsi repris la tradition du mawsim et lui annoncer que la colonie de Halamish sera bientôt détruite. En islam l'invention d'un lieu de culte est généralement précédée par l'apparition en songe d'un saint ou d'un prophète qui demande qu'une tombe lui soit construite et que des visites pieuses lui soient rendues (39). L'authenticité d'une tombe de saint ou de prophète n'étant jamais définitivement acquise, elle doit sans cesse être réaffirmée par de nouveaux miracles et apparitions. L'homme de sciences, Nimr Sirhan se fait donc le continuateur de cette tradition et annonce un miracle prochain, la disparition de la colonie voisine.

À Nabî Mûsâ, on retrouve également l'emprise d'un clan familial sur le mawsim et un rêve fondateur. La prestigieuse famille de Jérusalem, les Husaynî, assurait le patronage des festivités au début du XX $\mathrm{X}^{\mathrm{e}}$ siècle. D'après la tradition c'est un de leurs ancêtres, Cheikh 'Abdallah b. al-Yûnis qui, après que Moïse lui est apparu en rêve, ordonna la construction du sanctuaire et la tenue d'un pèlerinage annuel sur sa tombe. Cet homme n'était autre qu'un guerrier de l'armée de Salâh ad-Dîn. Ainsi, comme pour Nabî Sâlih, on retrouve le triptyque ancêtre (Cheikh 'Abdallah) - héros (Salâh ad-Dîn) - prophète (Moïse). Cela dit, il existe une grande différence entre les deux prophètes : Moïse, contrairement à Sâlih, a une identité précise. Pendant le mawsim les grands événements de sa vie mêlés à ceux de l'histoire du Prophète Muhammad sont racontés aux pèlerins dans la mosquée. Pas un seul fidèle qui ne connaisse les détails de la mission divine du Grand Législateur et des épreuves qu'il traversa. Même ses caractéristiques physiques sont connues de tous: immense, barbu, la peau noire, le visage entouré d'un halo lumineux et habillé de blanc. Le prophète Sâlih au contraire n'est pas un individu personnalisé et historicisé. Le récit coranique le concernant est court et énigmatique. Même s'il est largement complété par la littérature des Contes édifiants sur les prophètes (qisâs al-anbiyâ'), dans la pratique, son identité reste floue et labile.

(38) Deux articles parus dans Al-Ayyâm en avril et mai 1997 intitulés « Fî-1-manâm, zâranî an-Nabî Sâlih ».

(39) Catherine Mayeur-JaOuen note que la seule échappatoire à la dictature du lieu est le rêve, op. cit. p. 140 . 
Sâlih, personnage coranique non identifiable dans la Bible, fait partie des prophètes « arabes » (avec Hûd, Shu'ayb et Muhammad). Selon le Coran, il fut envoyé dans le Hedjaz auprès du peuple de Thamûd (40). Le symbole et l'essence de son message étaient une chamelle (nâqa) qui donnait du lait en abondance. Le peuple de Thamûd, au lieu de l'abreuver comme Dieu le lui avait demandé, lui coupa les jarrets et l'égorgea. Le châtiment divin descendit sur eux sous forme d'un tremblement de terre (41). Voici pour le récit coranique. Dans la pratique, le prophète arabe antéislamique mentionné dans les textes disparaît souvent pour devenir un guerrier, un saint ou encore un ancêtre. Nabî Sâlih se prête à toutes les transfigurations. Je l'ai déjà mentionné plus haut, il est souvent présenté comme un guerrier de Salâh ad-Dîn. À l'entrée de son sanctuaire à Ramleh, sur un panneau du ministère israélien des Antiquités il est expliqué que «Nabî Salih a servi dans l'armée de Salâh ad-Dîn ». Prophète-mujâhid, il est également assimilé à un saint (rajûl sâlih) ou encore à un ancêtre. Dans les discours, il est fréquent d'entendre des fidèles (surtout des villageois) l'appeler « cheikh Sâlih ». Ses identités multiples restent floues - elles se réduisent à un seul nom «Sâlih » (42) - et constituent autant d'espaces possibles pour l'apparition de nouvelles figures à ses côtés : celle de l'ancêtre du clan des Tamîmî, Tamîm ad-Dârî et celle du grand héros de l'histoire musulmane, Salâh ad-Dîn (43).

Le prophète dont la présence dans le village est symbolisée par le cénotaphe a joué un rôle essentiel dans l'ancrage du mawsim dans son nouveau cadre. Mais son sanctuaire cristallise de multiples tensions. Tout d'abord une tension qui ne disparaîtra jamais tout à fait entre le lieu saint originel et le mawsim réinventé. Tension à laquelle s'ajoute celle toujours latente entre une certaine orthodoxie qui rejette le culte de saints et une tradition élevée au rang de pierre angulaire de l'identité palestinienne. Enfin, il existe une troisième tension importante entre pratique rituelle individuelle et culte collectif. Le sanctuaire de celui que les villageois nomment souvent « cheikh Sâlih », est un lieu de culte privé. Ceci m'a été révélé au cours d'un de mes séjours dans le village. Je désirais me rendre dans le sanctuaire mais la femme qui m'hébergeait refusait de m'y accompagner, remettant toujours à plus tard notre visite. Un jour alors que je me faisais insistante, une de ses voisines présente me dit en riant : « Mais qu'as-tu ? Tu es malade ? Quelqu'un est malade chez toi ? Tu veux faire un nidhr (vœu) ? Vas d'abord chez le docteur et après on verra si

(40) Le peuple de Thamûd a souvent été identifié avec les Nabatéens, dont la capitale était Pétra en Jordanie.

(41) Coran : VII, 73-79 ; XI, 61-68, 89 ; XXVI, 141-158; XXVII, 45-53 ; XCI, 11-15.

(42) Sur l'importance du nom des saints musulmans dans le culte qui leur est rendu, cf. Catherine Mayeur, "L'intercession des saints en Islam égyptien ; autour de Sayyid al-Badawî », Annales islamologiques, $\mathrm{n}^{0} 25,1991$, pp. 363-388. Ainsi que ces quelques lignes d'Alphonse Dupront à propos des saints de l'Europe chrétienne : "Retient-on à suffisance que pris dans son évolution temporelle le culte des saints devient essentiellement invocation, voire évocation d'un nom ? » Sa légende peut disparaître. « Le nom garde seul toute sa puissance de communication. C'est pour lui, à travers lui, que se fixe, dans le mental populaire, la personne du saint. Qu'importe ce qu'il fut ; par le nom il est là ", Du sacré. Croisades et pèlerinages, images et langages, Paris, Gallimard, 1987 (coll. « NRF »).

(43) Sur la polysémie de Nabî Sâlih, cf. Emmanuel Marx qui dans son article sur les pèlerinages bédouins du Sinaï évoque l'existence d'un sanctuaire important dédié à Nabî Sâlih dans cette région. Dans une partie réservée à la signification symbolique des saints, il note la double identité de Nabî Sâlih à la fois revendiqué comme ancêtre par la tribu des Sawalha et assimilé au prophète coranique. "Tribal Pilgrimages to Saint's Tombs in South Sinai » in Ernest Gellner, ed., Islamic Dilemmas; Reformers, Nationalists and Industrialization, New York, Mouton, 1985. pp. 104-127. 
on t'emmène chez Nabî Sâlih. » Par ces mots elle m'a fait comprendre que ce lieu concentrait toute l'intimité du village, le lieu où les femmes en souffrance, « dos au mur » se rendent pour prier, invoquer le prophète.

Le sanctuaire de Nabî Sâlih est un lieu fermé qui renferme les secrets du village (femmes en souffrance) ; un lieu où « le vrai Nabî Sâlih » (maqâm de Ramleh) se rappelle au souvenir des fidèles; un lieu où se révèle le conflit latent entre orthodoxie et rituels populaires. Toutes ces raisons permettent de comprendre pourquoi l'ensemble des acteurs du pèlerinage (organisateurs, visiteurs et villageois) ont mis à l'écart le sanctuaire des festivités.

\subsection{Marginalisation du sanctuaire, multiplication des lieux symboliques}

Depuis le début de la nouvelle Intifada tous les mawsim-s palestiniens ont été annulés. Aussi, la fête telle qu'elle a été ressuscitée par l'Autorité palestinienne n'a eu lieu que quatre fois, entre 1997 et 2000. Sur cette période extrêmement courte, d'une année sur l'autre elle a pris des formes sensiblement différentes. Une tendance nette pouvait être dégagée : la disparition progressive du contenu religieux des cérémonies et l'imposition massive de symboles nationalistes. Un exemple frappant était l'appellation qui s'imposait peu à peu. En 2000, dans la presse on parlait de moins en moins de mawsim et de plus en plus de mahrajân watan̂̀ (festival national) et de carnaval. Les confréries soufies déjà peu nombreuses au début (3 en 1998) étaient totalement absentes la dernière année. La troupe de chanteurs mystiques de Jérusalem était la seule à avoir accepté de faire le déplacement en 2000 mais s'était retirée de la grande procession du premier jour parce que les organisateurs la plaçaient après les scouts. Ces derniers, avec leurs drapeaux palestiniens, leurs kéfiehs, leurs chants militaires et leurs hymnes étaient de plus en plus à l'honneur, et suscitaient une grande émotion chez les visiteurs. Les deux premières années, le mawsim débutait le vendredi juste après la grande prière, les années suivantes les organisateurs préféraient ouvrir les festivités le jeudi, veille du week-end, juste après la fermeture des bureaux. Surtout, le sanctuaire du prophète Sâlih qui avait permis l'ancrage du mawsim dans le village était d'année en année un peu plus délaissé. Sa marginalisation a été définitive en 1998, lorsque trois autres lieux hautement symboliques ont été construits dans le village.

Pour pouvoir accueillir les visiteurs qui l'envahissaient à l'occasion du mawsim, le village avait subi de grandes transformations. Devenu symbole de l'identité palestinienne il se devait d'être aussi resplendissant que possible et c'est dans sa totalité - avec ses champs d'oliviers et ses vieilles maisons de pierre - qu'il était célébré. L'entrée principale du village a été déplacée, la route empruntée par la procession le premier jour des festivités a été asphaltée et une centaine de structures permanentes ont été mises en place dans le village pour abriter les échoppes des commerçants qui venaient vendre leurs produits pendant le mawsim. Surtout, trois nouveaux lieux symboliques ont été construits (un immense théâtre, un monument aux morts et le diwân des Tamîmî) qui constituaient autant de centres où les visiteurs étaient invités à converger pour célébrer la mémoire et la nation palestiniennes.

Le seul lieu qui n'ait pas été restauré dans le village est le sanctuaire lui-même. La petite qubba accolée à une mosquée est restée délabrée et la route qui y mène 
n'a pas été asphaltée. Pour y accéder, il faut emprunter une petite piste de terre sur quelques mètres et passer par un escalier étroit et dangereux. Les deux premières années, il constituait le point autour duquel les festivités se déroulaient. La grande procession du premier jour se formait après la prière du vendredi devant son entrée et, après avoir fait le tour du village, y revenait. Les spectacles se déroulaient sur la petite place située en face de la qubba. Après la construction du monument aux morts, du diwân des Tamîmî et du théâtre, c'est un tout autre parcours qui était suivi par la grande procession. Parcours où le sanctuaire constituait une étape facultative.

\subsection{Le patrimoine revivifié et exalté}

Le nombre de visiteurs atteignait le chiffre de 20000 certains jours. Le public était varié : des familles venues des villages alentours croisaient des habitants des principales villes de Cisjordanie et des groupes d'étudiants (hommes et femmes mélangés, chrétiens et musulmans) de l'université de Bir Zeit toute proche.

Le théâtre ouvert pouvait accueillir un large public et constituait le véritable objectif des visiteurs qui étaient nombreux à s'installer pour la journée dans ses alentours avec nattes, coussins et réchauds à gaz. Les spectacles commençaient à 14 heures et s'achevaient à 18 heures. La volonté des organisateurs était de représenter l'ensemble de la Palestine à travers ces spectacles. Chaque journée était consacrée à une région particulière ; le premier jour était réservé à Ramallah et ses environs, le second à Naplouse, le troisième à Jéricho, le quatrième à Hébron et le cinquième, au «territoire de 1948 » (44). Entre chaque représentation, des représentants de l'Autorité palestinienne prononçaient des discours politiques. Pour certains spectacles, les rues, les maisons et les échoppes du village se vidaient entièrement et l'immense esplanade pouvait à peine contenir l'afflux massif des spectateurs. Par contraste, la petite place devant la qubba de Nabî Sâlih semblait bien vide. En 2000, le directeur du Comité y a même autorisé l'installation d'un petit circuit d'autos-tamponneuses. Cette année là, les seuls à répondre à l'appel du muezzin pour la prière du vendredi étaient les habitués du village rejoints par quelques autres fidèles, généralement des habitants des villages alentours.

Deux mots revenaient systématiquement dans les discours des organisateurs et des visiteurs sur le mawsim : « renouveau » (ihyâ') et "patrimoine » (turâth). Le mot ihyâ' était chaque année inscrit sur les affiches invitant à participer aux festivités. Dans le titre choisi pour désigner le comité organisateur - «Haut comité pour la reprise, le renouveau du mawsim de Nabî Sâlih »- cette notion était soulignée avec une certaine redondance. Lorsque je demandais sa signification, l'accent était mis sur l'idée du renouveau et surtout pas de la répétition littérale d'une ancienne tradition. La tradition exhumée du passé devait être adaptée aux nécessités du présent, devenir 'moderne' : «Je sais bien que tu voudrais nous voir porter des habits traditionnels, que tu voudrais nous voir danser et chanter le nom de Dieu comme font les Égyptiens en bougeant la tête dans tous les sens! Mais non! Le mawsim est devenu une fête moderne comme votre carnaval en Europe », m'expliquait le

(44) Expression employée pour désigner les Palestiniens de nationalité israélienne. 
maire du village, 'Abdel Rahmân Tamîmî. Le patrimoine n'en demeurait pas moins exalté. Il était partout présent dans le village : dans les spectacles de danses et de chants traditionnels; dans les robes brodées des paysannes; dans les objets vendus dans les échoppes ; dans la nourriture offerte ; dans la pierre des vieilles maisons, dans les champs d'olivier; dans le four traditionnel d'Um Ahmad. À travers le turâth exalté c'est la Palestine enfin réunie qui était célébrée. Les différents spectacles étaient censés symboliser la victoire d'une Palestine retrouvée et rassemblée (45).

\subsection{La lutte pour la terre : la procession et le bruit}

Mais dans le même moment le village symbolisait une lutte toujours en cours et loin d'être gagnée : le combat contre la colonisation. Envahir les ruelles et les champs alentours, c'était affirmer sa résistance et son attachement à la terre. Le pèlerinage constituait une forme de réappropriation et d'occupation de l'espace. La séquence importante du voyage vers le village était toujours largement commentée par les visiteurs et les organisateurs. Un des sujets de conversation portait sur le parcours - sa longueur, les barrages militaires, les villages traversés, l'extension des colonies... - emprunté par les uns et les autres pour venir participer au mawsim. Les organisateurs commentaient "la guerre des panneaux» qui chaque année s'engageait avec les colons de Halamish et l'armée israélienne. Le chemin vers le village, comme la plupart des localités palestiniennes de Cisjordanie, n'est pas signalé. Pour y accéder il faut suivre les panneaux indiquant la colonie. Quelques jours avant le début des festivités, les organisateurs plantaient le long de la route des panneaux «Nabî Sâlih» qui étaient presque immédiatement arrachés par les colons et replantés par les membres du Comité du pèlerinage pour être de nouveau arrachés...

Un autre moment important du mawsim était constitué par la procession. Les visiteurs n'étaient pas tenus de se plier à une pratique rituelle fixe. Ils étaient libres de déambuler dans les rues du village pour profiter du marché provisoire, d'envahir les champs d'oliviers alentours pour y pique-niquer, d'assister aux spectacles qui se déroulaient dans le théâtre, de se rendre dans le sanctuaire pour prier. Seule la grande procession du premier jour avait un caractère obligatoire. Elle mobilisait l'ensemble des acteurs. Elle était organisée à partir de la maison du président du conseil villageois, avec en tête les personnalités politiques suivies de la fanfare militaire, des scouts et du groupe de chanteur mystique de Jérusalem. Une chamelle blanche, censée représenter la nâqa du prophète Sâlih ouvrait la marche. Elle était montée par un homme en habit militaire qui tenait le drapeau palestinien. La longue file progressait lentement vers le centre du village. Elle faisait un arrêt devant le monument aux morts du village. La Fâtiha était lue, le drapeau palestinien était solennellement levé et après une minute de silence pour les martyrs palestiniens, elle repartait vers le sanctuaire de Nabî Sâlih où seuls quelques hommes entraient

(45) Sur le mélange de tradition et modernité dans le contexte de la construction nationale ; sur le renouveau et la mobilisation d'un rituel ancien dans un objectif politique et nationaliste, cf. Vittorio LANTERNARI, The Religions of the Oppressed. A Study of Modern Messianic Cults, Londres, MacGibbon and Koe, 1965. 
pour une visite pieuse qui ne consistait qu'en une simple lecture de la Fâtiha. Puis la colonne se reformait et se dirigeait vers le théâtre situé à l'autre bout du village. Ce défilé mené par les politiques au rythme des chants nationalistes traversait de part en part le village qui était ainsi dans son entier occupé et célébré.

Contre l'ennemi colon, si proche mais invisible, le bruit également jouait un rôle essentiel. Des haut-parleurs étaient disposés un peu partout à l'intérieur et autour du village. Dispositif qui rendait le niveau sonore insupportable pendant les spectacles et la grande procession et qui était destiné à atteindre la colonie. En 1999, à la grande joie des villageois, la colonie a tenté des représailles en faisant également un tapage inouï avec des haut-parleurs qui retransmettaient l'hymne national israélien et des chants religieux juifs.

\section{Conclusion}

Le déplacement du mawsim de la ville de Ramleh dans le village de Nabî Sâlih s'est fait au détriment de sa signification religieuse et avec un renforcement de son caractère politique et nationaliste. La situation géographique particulière du village, la proximité d'une colonie, les confiscations de terres dont il est régulièrement victime, l'administration partielle exercée par l'autorité confère au mawsim « ressuscité » son caractère nationaliste. Finalement, c'est le lieu et à travers lui le combat pour la construction nationale et non le prophète que l'on venait célébrer.

Depuis le début de l'Intifada, le mawsim n'a plus lieu. La " fête est terminée » me disait un villageois, "c'est la guerre!». Le mawsim tel qu'il a été réinventé par l'Autorité palestinienne se raconte au passé et laisse un souvenir amer chez beaucoup, le sentiment d'avoir prématurément fêté la construction nationale et d'avoir trahi, d'avoir détourné le rituel du mawsim de la religion : "Ils ont construit un théâtre mais c'est une vraie grande mosquée qu'ils auraient dû construire » me disait une femme «nous ne pouvons pas continuer comme cela, organiser des fêtes où les hommes et les femmes dansent ensemble la dabka. Ils disent que la dabka est notre turâth. Non, notre turâth c'est la religion. »

Avant l'Intifada, le village constituait un lieu symbolique construit et reconstruit chaque année par l'action conjuguée des visiteurs et des organisateurs pendant le temps du mawsim. Il symbolisait tout à la fois la construction nationale et la lutte contre la colonisation. Avec l'Intifada, tout cela a disparu : le village soudainement banalisé a perdu sa fonction centrale. Peu a peu le lieu entre guillemets, «Nabî Sâlih » (46), s'est délité ; le théâtre trop éloigné du village s'est recouvert de mauvaises herbes; le monument aux morts a perdu de sa superbe ; la route principale a été détruite... " Le village palestinien » a disparu pour laisser place à " un » petit village palestinien avec ses particularités.

(46) Sur la construction des lieux entre guillemets, cf. Elisabeth CLAVERIE « Apparition de la vierge et "retour" des disparus », Terrain, no 38 , mars 2002, pp. 41-54. 
Dans le paysage de désolation qu'il offre aujourd'hui, la qubba du prophète Sâlih a retrouvé sa place centrale. Bien plus, il n'a pas suivi le mouvement de délitescence générale ; il demeure inchangé malgré les bouleversements. Les personnes âgées du village continuent de s'y retrouver le matin avant la prière de midi; les femmes y rendent toujours des visites pieuses discrètes. La situation de guerre qui prévaut depuis deux ans m'a permis de mieux comprendre les raisons de la marginalisation du sanctuaire pendant le mawsim. Elle ne doit pas être uniquement comprise comme le signe d'une sécularisation inexorable du mawsim mais surtout comme la volonté des habitants de Nabî Sâlih de préserver un lieu "privé » et intime des flots de visiteurs qui envahissent le village.

Le mawsim réinventé, vidé de sa signification religieuse s'est révélé d'une grande fragilité. Il n'a plus lieu et ne reprendra probablement pas. Et ceci contrairement au mawsim de Nabî Mûsâ qui aujourd'hui encore, malgré les événements, continue d'avoir lieu à une très petite échelle. Il s'agit essentiellement de groupes de femmes qui viennent pour faire un vœu pour un proche malade.... Là également l'Autorité palestinienne avait tenté de contrôler les festivités en établissant un programme. Mais contrairement à Nabî Sâlih les organisateurs perdaient très vite le contrôle des événements. Notamment, l'interdiction qui était faite aux femmes de se rendre dans la salle abritant le cénotaphe sous prétexte que la mosquée était réservée aux hommes et qu'une autre salle de prière avait été aménagée pour les femmes, n'était pas respectée. Le tombeau de Moïse était resté central et les tensions inévitables qu'il cristallisait n'ont pu être évitées. Le mawsim se déroulait dans une atmosphère d'anarchie et de dérégulation. Mais dans le même temps le lien particulier entre les fidèles, le prophète et Dieu qui s'établissait à travers les rituels entourant le tombeau n'était pas coupé et il semble que, contrairement aux cérémonies d'exaltation de la nation, il soit essentiel à la pérennité du rituel du mawsim.

\author{
Emma AUBIN BOLTANSKI
} EHESS/IISMM - Paris 
Résumé

Entre 1997 et 2000, les territoires palestiniens sont le théâtre d'une fragile construction nationale débutée trois ans plus tôt. Ce processus est brutalement interrompu par l'éclatement de l'Intifada al-Aqsa. Pendant cette courte période, l'Autorité palestinienne, par l'intermédiaire de ses ministères des Affaires religieuses et de la Culture, s'est impliquée dans l'organisation de deux pèlerinages musulmans : les mawsim-s de Nabî Mûsâ et de Nabî Sâlih. Ces deux fêtes religieuses sont l'occasion pour les dévots de nouer des liens intimes et à chaque fois particuliers avec les prophètes et, à travers eux, avec Dieu. Elles sont également des grands moments d'exaltation nationaliste. Ce dernier caractère sera considérablement renforcé par l'Autorité palestinienne. L'objet de cet article sera d'analyser les transformations qui ont tout particulièrement affecté le mawsim de Nabî Sâlih. Il sera démontré que des sanctuaires tout en se caractérisant par une grande immobilité sont habités par une tradition sans cesse remaniée, reconstruite et que des tensions peuvent s'établir entre les lieux saints et la tradition éminemment plastique qui se fixe sur eux.

\section{Abstract}

Between 1997 and 2000, the Palestinian territories saw a fragile state building process, which had started three years earlier. The outbreak of the Intifada al-Aqsa in October 2000 brought it to a brutal end. During this short period of time, the Palestinian Authority, through the intermediary of the Ministry of Religious Affairs and the Ministry of Culture was involved in the organisation of two Muslim pilgrimages : the mawsim-s of Nabî Mûsâ and Nabî Sâlih. During these two religious festivals, worshipers create intimate and individual ties with the prophets and through them with God. They are also great occasions for national glorification. This last characteristic was considerably reinforced by the Palestinian Authority. This article will closely analyse the transformations which have particularly affected the mawsim of Nabî Sâlih. It will demonstrate how the sanctuaries, whilst being characterized by a great immobility are inhabited by an unceasingly altered and rebuilt tradition, and that tensions can arise between these holy places and the eminently plastic tradition which is fixed upon them.

\section{Resumen}

Entre 1997 y 2000, los territorios palestinos son el escenario de una frágil construcción nacional comenzada tres años más temprano. Este proceso es brutalmente interrumpido por el estallido de la Intifada al-Aqsa. Durante este corto período, la Autoridad palestina, por medio de sus ministros de Asuntos religiosos y de Cultura, se implicó en la organización de dos peregrinaciones musulmanas: los mawsim-s de Nabî Musa y de Nabî Sâlih. Estas dos fiestas religiosas son la ocasión para los fieles de establecer lazos íntimos, y cada vez particulares, con los profetas y, a través de ellos, con Dios. Son también grandes momentos de exaltación nacionalista. Este último carácter será considerablemente reforzado por la Autoridad palestina. El objetivo de este artículo es analizar las transformaciones que afectaron particularmente el mawsim de Nabî Sâlih. Se demostrará que santuarios, aún caracterizándose por una gran inmovilidad, son habitados por una tradición reformulada sin cesar, reconstruida, y que diversas tensiones pueden establecerse entre los lugares santos y la tradición eminentemente plástica que se fija sobre ellos. 